\title{
PYTHAGOREAN IDENTITY FOR POLYHARMONIC POLYNOMIALS
}

\section{ALLAN FRYANT and MURALI KRISHNA VEMURI}

Received 13 May 1999

Polyharmonic polynomials in $n$ variables are shown to satisfy a Pythagorean identity on the unit hypersphere. Application is made to establish the convergence of series of polyharmonic polynomials.

2000 Mathematics Subject Classification: 31B99.

1. Introduction. Let $L_{n}^{k}$ denote the vector space of real homogeneous polynomial solutions of degree $k$ of Laplace's equation

$$
\Delta u=0,
$$

where

$$
\Delta=\frac{\partial^{2}}{\partial x_{1}^{2}}+\frac{\partial^{2}}{\partial x_{2}^{2}}+\cdots+\frac{\partial^{2}}{\partial x_{n}^{2}} .
$$

Such polynomials are called spherical harmonics. As shown in [9, pages 140-141],

$$
\operatorname{dim} L_{n}^{k}=d_{n}^{k}=(n+k-2) \frac{(n+2 k-3) !}{k !(n-2) !} .
$$

Suppose that $\left\{y_{j}^{k}(x)\right\}_{j=1}^{d_{n}^{k}}$ is an orthonormal basis for $L_{n}^{k}$, where orthonormality is with respect to the inner product

$$
\langle f, g\rangle=\int_{\sum_{1}} f(x) g(x) d x
$$

on the unit sphere $\sum_{1}: x_{1}^{2}+x_{2}^{2}+\cdots+x_{n}^{2}=1$. It is well known (cf. [9, page 144]) that for all $s \in \sum_{1}$,

$$
\sum_{j=1}^{d_{n}^{k}}\left[y_{k}^{j}(s)\right]^{2}=\omega_{n} d_{n}^{k},
$$

where $\omega_{n}$ is the surface area of the unit sphere $\sum_{1}$ in $\mathbb{R}^{n}$. We call (1.5) the Pythagorean identity for spherical harmonics, since it generalizes the Pythagorean theorem

$$
\sin ^{2} \theta+\cos ^{2} \theta=1 \text {. }
$$

Solutions of partial differential equation

$$
\Delta^{m} u=0
$$


where $\Delta$ is the Laplacian (1.2) and $m$ is a positive integer, are called polyharmonic functions. In the case $m=2$, such functions are called biharmonic and are used to model the bending of thin plates (for a brief history of this application, see [7, pages 416 and 432-443]).

We show here that homogeneous polyharmonic polynomials satisfy a Pythagorean identity on $\sum_{1}$ and use this identity to establish the convergence of polyharmonic polynomial series.

2. Pythagorean identity. Let $J_{n}^{k}$ denote the vector space of real homogeneous polynomial solutions of the partial differential equation (1.7). Since $\Delta^{m}$ is a homogeneous differential operator of order $2 m$, using a standard argument (cf. [5, Theorem 1]) we find that

$$
\operatorname{dim} J_{n}^{K}=b_{n}^{k}=\left(\begin{array}{c}
n-1+k \\
k
\end{array}\right)-\left(\begin{array}{c}
n-1+k-2 m \\
k-2 m
\end{array}\right) .
$$

In the vector space $J_{n}^{k}$, we introduce the Calderón inner product [1]

$$
(p, q)=p\left(\frac{\partial}{\partial x}\right) q(x)
$$

where

$$
\frac{\partial}{\partial x}=\left(\frac{\partial}{\partial x_{1}}, \frac{\partial}{\partial x_{2}}, \ldots, \frac{\partial}{\partial x_{n}}\right), \quad p\left(\frac{\partial}{\partial x}\right)=p\left(\frac{\partial}{\partial x_{1}}, \frac{\partial}{\partial x_{2}}, \ldots, \frac{\partial}{\partial x_{n}}\right)
$$

THEOREM 2.1. Suppose that $\left\{Q_{k}^{j}(x)\right\}_{j=1}^{b_{n}^{k}}$ is an orthonormal basis for the vector space $J_{n}^{k}$ of homogeneous polyharmonic polynomials of degree $k$, where orthonormality is with respect to the inner product (2.2). Then for all $s=\left(s_{1}, s_{2}, \ldots, s_{n}\right) \in \sum_{1}$, the unit sphere in $\mathbb{R}^{n}$,

$$
\sum_{j=1}^{b_{n}^{k}}\left[Q_{k}^{j}(s)\right]^{2}=\gamma_{n}^{k}
$$

where $\gamma_{n}^{k}$ is a constant depending only on $n$ and $k$.

Proof. A modification in the argument used for spherical harmonics suffices: fix a point $y \in \mathbb{R}^{n}$ and consider the linear functional $L: J_{n}^{k} \rightarrow \mathbb{R}$ defined by

$$
L(p)=p(y) .
$$

Since $J_{n}^{K}$ is a finite-dimensional inner product space, there exists a unique $Z_{y} \in J_{n}^{k}$ such that

$$
L(p)=\left(p(x), Z_{y}(x)\right)
$$

for all $p \in J_{n}^{k}$ (i.e., all finite-dimensional inner product spaces are self-dual). Further, since $\left\{Q_{k}^{j}(x)\right\}_{j=1}^{b_{n}^{k}}$ is an orthonormal basis for $J_{n}^{k}$,

$$
Z_{y}(x)=\sum_{j=1}^{b_{n}^{k}}\left(Z_{y}(x), Q_{k}^{j}(x)\right) Q_{k}^{j}(x) .
$$


But, by the defining property of $Z_{y}$,

$$
\left(Z_{y}(x), Q_{k}^{j}(x)\right)=Q_{k}^{j}(y)
$$

Hence

$$
Z_{y}(x)=\sum_{j=1}^{b_{n}^{k}} Q_{k}^{j}(y) Q_{k}^{j}(x) .
$$

Since the choice of $y \in \mathbb{R}^{n}$ was arbitrary, $z_{y}(x)$ is a function of the two variables $x, y \in \mathbb{R}^{n} ;$ thus, we write

$$
Z(x, y)=Z_{y}(x)=\sum_{j=1}^{b_{n}^{k}} Q_{k}^{j}(x) Q_{k}^{j}(y) .
$$

The Calderón inner product (2.2) is invariant with respect to rotations; that is, if $O: \mathbb{R}^{n} \rightarrow \mathbb{R}^{n}$ is a rotation, then $(f(x), g(O x))=\left(f\left(O^{-1} x\right), g(x)\right)$. Suppose $p(x) \in J_{n}^{k}$. Then

$$
(p(x), Z(O x, O y))=\left(p\left(O^{-1} x\right), Z(x, O y)\right)=(q(x), Z(x, O y)),
$$

where $q(x)=p\left(O^{-1} x\right)$. Since rotations are invariant transformations for the Laplacian, it follows that $q(x) \in J_{n}^{k}$. Thus, by the defining property of $Z(x, y)$,

$$
(q(x), Z(x, O y))=q(O y) .
$$

But $q(O y)=p\left(O^{-1} O y\right)=p(y)$. Thus, we have shown that

$$
(p(x), Z(O x, O y))=p(y) .
$$

From the uniqueness of the representation of linear functionals, it follows that

$$
Z(O x, O y)=Z(x, y)
$$

for all $x, y \in \mathbb{R}^{n}$. In particular,

$$
Z(O x, O x)=Z(x, x)
$$

for every rotation $O$. Since every point on the unit sphere $\sum_{1}$ is the image under rotation for some fixed point on $\sum_{1}$, the equality (2.15) implies that $Z(x, x)$ is constant on $\sum_{1}$. That is,

$$
\sum_{j=1}^{b_{n}^{k}} Q_{k}^{j}(s) Q_{k}^{j}(s)=C
$$

a constant, for all $s \in \sum_{1}$.

3. Polyharmonic polynomial series. Pythagorean identities have been used to establish the convergence of series of spherical harmonics [4], as well as series of orthonormal homogeneous polynomials in several real variables in general [3]. We obtain here convergence for series of polyharmonic polynomials. 
THEOREM 3.1. Suppose that $\left\{Q_{k}^{j}(x)\right\}_{j=1}^{b_{n}^{k}}$ are sets of orthonormal polyharmonic polynomials in $\mathbb{R}^{n}$ of degree $k, k=0,1,2, \ldots$. Then the series

$$
\sum_{k=0}^{\infty} \sum_{j=1}^{b_{n}^{k}} a_{k j} Q_{k}^{j}(x)
$$

converges absolutely and uniformly on compact subsets of the open ball $|x|=\left(x_{1}^{2}+\right.$ $\left.x_{2}^{2}+\cdots+x_{n}^{2}\right)^{1 / 2}<R$, where

$$
R^{-1}=\limsup _{k \rightarrow \infty}\left(\sqrt{\gamma_{n}^{k}}\left\|a_{k}\right\|\right)^{1 / k}, \quad\left\|a_{k}\right\|=\left(\sum_{j=1}^{b_{n}^{k}} a_{k j}^{2}\right)^{1 / 2},
$$

and $\gamma_{n}^{k}$ is the Pythagorean constant appearing in (2.4).

Proof. Since each of the polynomials $Q_{k}^{j}$ is homogeneous of degree $k$, we have $Q_{k}^{j}(x)=r^{k} Q_{k}^{j}(x / r)$, where $r=\left(x_{1}^{2}+x_{2}^{2}+\cdots+x_{n}^{2}\right)^{1 / 2}$. Thus

$$
\begin{aligned}
\left|\sum_{k=0}^{\infty} \sum_{j=1}^{b_{n}^{k}} a_{k j} Q_{k}^{j}(x)\right| & =\left|\sum_{k=0}^{\infty} r^{k} \sum_{j=1}^{b_{n}^{k}} a_{k j} Q_{k}^{j}\left(\frac{x}{r}\right)\right| \\
& \leq \sum_{k=0}^{\infty} r^{k}\left|\sum_{j=1}^{b_{n}^{k}} a_{k j} Q_{k}^{j}\left(\frac{x}{r}\right)\right|,
\end{aligned}
$$

by the Cauchy-Schwarz inequality

$$
\left|\sum_{k=0}^{\infty} \sum_{j=1}^{b_{n}^{k}} a_{k j} Q_{k}^{j}(x)\right| \leq \sum_{k=0}^{\infty} r^{k}\left(\sum_{j=1}^{b_{n}^{k}} a_{k j}^{2}\right)^{1 / 2}\left(\sum_{j=1}^{b_{n}^{k}} Q_{k}^{j}\left(\frac{x}{r}\right)\right)^{1 / 2} .
$$

Appealing now to the Pythagorean identity (2.4), we find that

$$
\left|\sum_{k=0}^{\infty} \sum_{j=1}^{b_{n}^{k}} a_{k j} Q_{k}^{j}(x)\right|=\sum_{k=0}^{\infty} r^{k}\left\|a_{k}\right\| \sqrt{\gamma_{n}^{k}}
$$

from which the desired result is immediate.

Let $H_{n}^{k}$ denote the vector space of homogeneous polynomials of degree $k$ in $\mathbb{R}^{n}$. Since every orthonormal basis of $J_{n}^{k}$ be extended to an orthonormal basis of $H_{n}^{k}$, it follows from [2, Theorem 3] that

$$
\gamma_{n}^{k} \leq \frac{1}{k !}
$$

Thus,

$$
R^{-1}=\limsup _{k \rightarrow \infty}\left(\sqrt{\gamma_{n}^{k}}\left\|a_{k}\right\|\right)^{1 / 2} \leq \limsup _{k \rightarrow \infty}\left(\frac{\left\|a_{k}\right\|}{\sqrt{k !}}\right)^{1 / k}=\rho^{-1},
$$


and appealing to the result of Theorem 3.1 we find that the polyharmonic polynomial series (3.1) converges absolutely and uniformly on compact subsets of the open ball $|x|<\rho$. We predict that the evaluation of the Pythagorean constant $\gamma_{n}^{k}$ will show that such convergence actually obtains within a somewhat larger ball.

In [11], it was shown that, in the space of homogeneous harmonic polynomials $L_{n}^{k}$, the Calderón inner product (2.2) is a constant multiple of the inner product (1.4). That is,

$$
(p, q)=c_{n}^{k}\langle p, q\rangle
$$

for all $p, q \in L_{n}^{k}$, where $c_{n}^{k}$ is a constant depending only on $n$ and $k$. Thus, the Pythagorean identity for spherical harmonics $(1.5)$ is a special case $(m=1)$ of the result of Theorem 2.1.

The Pythagorean identity for spherical harmonics is also a special case of the addition formula for spherical harmonics [9, page 149] and [8, page 268]. This leads us to conjecture that the homogeneous polyharmonic polynomials satisfy a similar addition formula, from which Theorem 2.1 might follow as an immediate consequence. Such a development could include a significant generalization of the ultraspherical polynomials $[6,10]$.

\section{REFERENCES}

[1] A. P. Calderón, Integrales singulares y sus aplicaciones a ecuaciones diferenciales hiperbolicas [Singular Integrals and their Applications to Hyperbolic Differential Equations], Cursos y Seminarios de Matemática, Fasc. 3. Universidad de Buenos Aires, Buenos Aires, 1960 (Spanish).

[2] A. Fryant, Multinomial expansions and the Pythagorean theorem, Proc. Amer. Math. Soc. 124 (1996), no. 7, 2001-2004.

[3] A. Fryant, A. Naftalevich, and M. K. Vemuri, Orthogonal homogeneous polynomials, Adv. in Appl. Math. 22 (1999), no. 3, 371-379.

[4] A. Fryant and H. Shankar, Bounds on the maximum modulus of harmonic functions, Math. Student 55 (1987), no. 2-4, 103-116 (1989).

[5] A. Fryant and M. K. Vemuri, Wave polynomials, Tamkang J. Math. 28 (1997), no. 3, 205209.

[6] A. J. Fryant, Ultraspherical expansions and pseudo analytic functions, Pacific J. Math. 94 (1981), no. 1, 83-105.

[7] V. Maz'ya and T. Shaposhnikova, Jacques Hadamard, a Universal Mathematician, History of Mathematics, vol. 14, American Mathematical Society, Rhode Island, 1998.

[8] G. Sansone, Orthogonal Functions, Pure and Applied Mathematics, vol. 9, Interscience Publishers, New York, 1959.

[9] E. M. Stein and G. Weiss, Introduction to Fourier Analysis on Euclidean Spaces, Princeton Mathematical Series, no. 32, Princeton University Press, New Jersey, 1971.

[10] G. Szegö, Orthogonal Polynomials, 3rd ed., American Mathematical Society Colloquium Publications, vol. 23, American Mathematical Society, Rhode Island, 1967.

[11] M. K. Vemuri, A simple proof of Fryant's theorem, SIAM J. Math. Anal. 26 (1995), no. 6, 1644-1646.

ALLAN FRYANT: 603F Simpson STREeT, GREENSBORO, NC 27401, USA

Murali Krishna Vemuri: Department of Mathematics, Syracuse UniVersity, SYracuse, NY 13244, USA

E-mail address: mkvemuri @math . syr . edu 


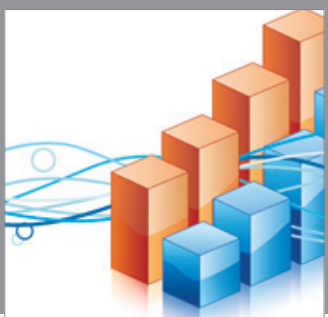

Advances in

Operations Research

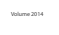

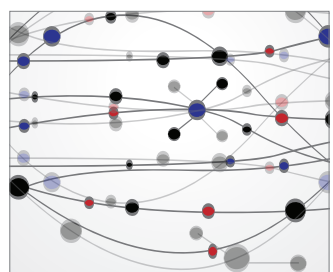

\section{The Scientific} World Journal
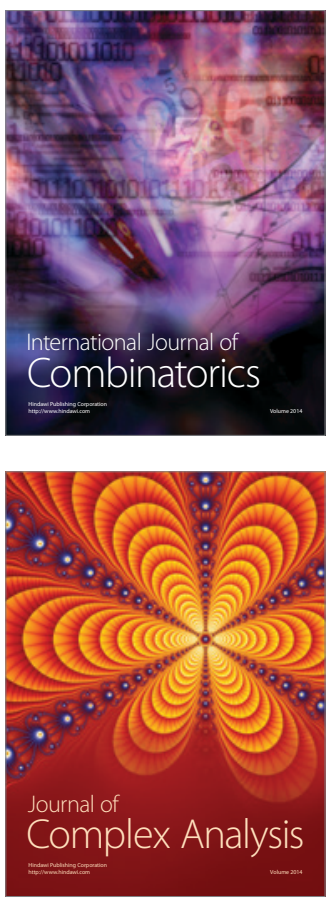

International Journal of

Mathematics and

Mathematical

Sciences
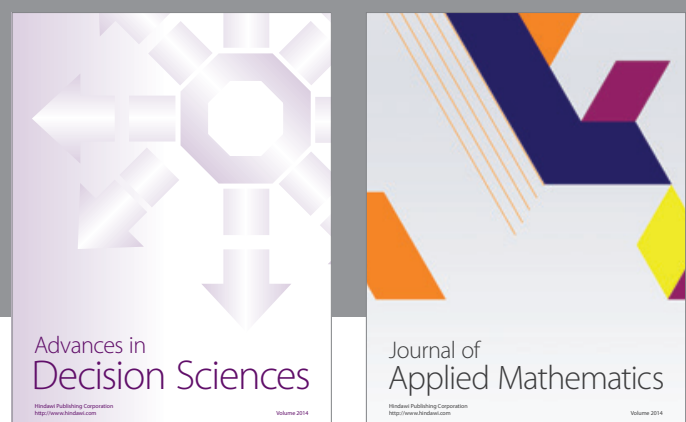

Journal of

Applied Mathematics
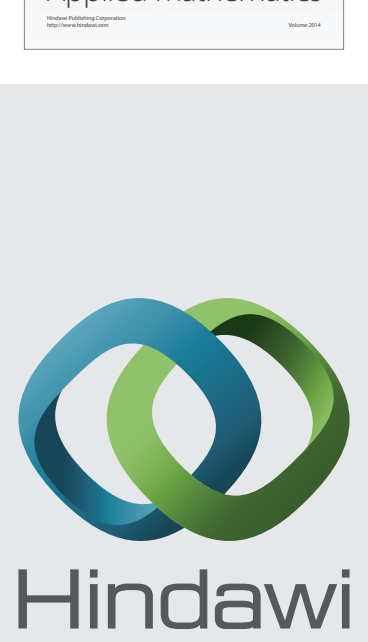

Submit your manuscripts at http://www.hindawi.com
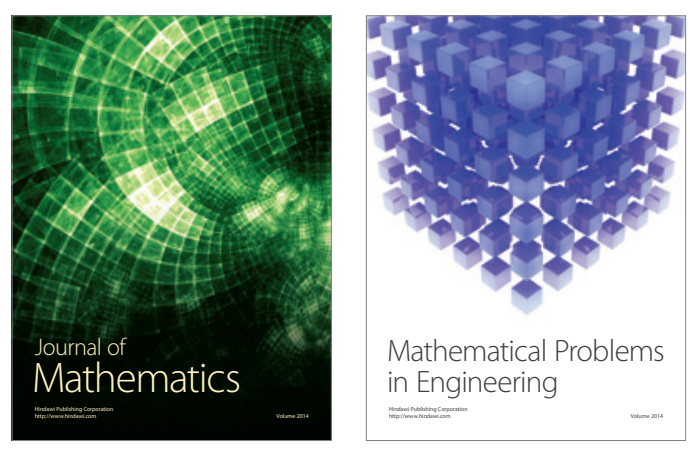

Mathematical Problems in Engineering
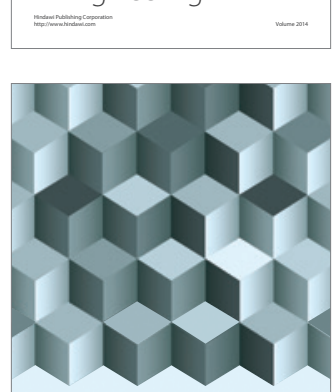

Journal of

Function Spaces
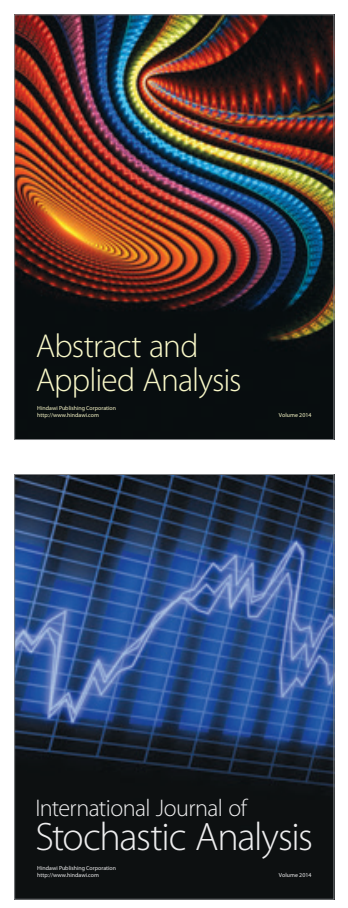

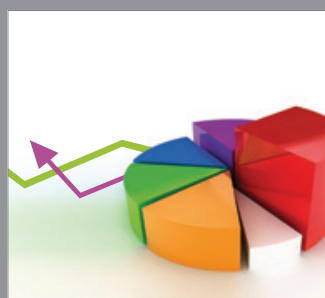

ournal of

Probability and Statistics

Promensencen
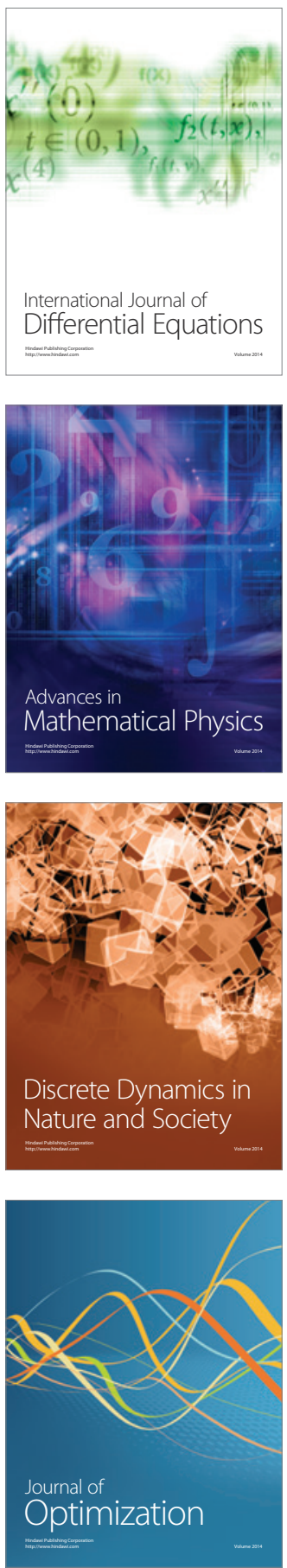